\title{
Approche procédurale de la lecture Catégories flottantes et unités élastiques
}

\author{
Lundquist, Lita Sander
}

Document Version

Final published version

Published in:

Semiotica

DOI:

10.1515/sem-2017-0022

Publication date:

2018

License

Unspecified

Citation for published version (APA):

Lundquist, L. S. (2018). Approche procédurale de la lecture: Catégories flottantes et unités élastiques.

Semiotica, (223), 141-152. https://doi.org/10.1515/sem-2017-0022

Link to publication in CBS Research Portal

\section{General rights}

Copyright and moral rights for the publications made accessible in the public portal are retained by the authors and/or other copyright owners and it is a condition of accessing publications that users recognise and abide by the legal requirements associated with these rights.

Take down policy

If you believe that this document breaches copyright please contact us (research.lib@cbs.dk) providing details, and we will remove access to the work immediately and investigate your claim. 


\section{Lita Lundquist*}

\section{Approche procédurale de la lecture : Catégories flottantes et unités élastiques}

https://doi.org/10.1515/sem-2017-0022

Résumé : L’article vise trois objectifs différents, mais intégrés : le français langue étrangère, la lecture et les textes spécialisés, trois objectifs qui nous situent au cœur de la linguistique textuelle contrastive, avec des excursions dans la linguistique cognitive et ses réflexions sur le rôle de différents types de savoir : savoir sur la langue, sur le texte et sur le monde. Dans la perspective procédurale de la lecture, nous considérons les catégories textuelles : discours, genre et type de texte comme des déclencheurs d'attentes " descendants ", et les unités linguistiques, à déterminer par leur forme, fonction et frontières, comme des déclencheurs « ascendants » du traitement « en ligne » du matériau linguistique arrivant. Nous illustrerons comment ces deux procédures, descendante et ascendante, se combinent dans une "lecture raisonnée », de prédiction-identification-vérification-(correction), où catégories textuelles et unités linguistiques se concrétisent et se déterminent en cours de route, tout selon le savoir linguistique, textuel et du monde, du lecteur.

Mots-clés : linguistique textuelle contrastive, français langue étrangère, textes spécialisés, lecture, perspective procédurale

\section{Introduction}

Tout comme la délimitation du concept de discours par rapport à celui de texte a fait couler beaucoup d'encre dans la théorie linguistique du texte - ou du discours ${ }^{1}$ - la catégorisation en unités et entités, et leur taxinomie, ne cesse d'intriguer les spécialistes. Nous situant dans un horizon de linguistique textuelle scandinave où la distinction entre texte et discours paraît surtout d'intérêt étymologique, texte étant dès les débuts de la linguistique du texte utilisé dans

1 Adam parle de «linguistique du texte et du discours » (Adam $2013:$ 303, 308).

*Corresponding author : Lita Lundquist, Copenhagen Business School, Frederiksberg, Denmark, E-mail : Il.dbp@cbs.dk 
la tradition germanique ${ }^{2}$ et discours plus répandu dans la tradition romaniste, ${ }^{3}$ avec la tradition anglo-saxonne hésitant entre les deux, ${ }^{4}$ nous allons ici brutalement couper court à ces discussions théoriques et attaquer la question des catégories d'analyse, unités et procédés par un biais proprement pratique.

Notre approche sera déterminée par trois objectifs différents, mais intégrés : le premier est un objectif didactique du français langue étrangère (FLE), lequel nous situe d'emblée dans une perspective contrastive, entre une L1 et une L2 (le cas échéant, le danois et le français). Le second est celui de la lecture, qui nous impose une optique à la fois textuelle et processuelle. Et le troisième est celui des textes spécialisés, ou « textes sur objet spécifique », ${ }^{5}$ objectif qui met en cause non pas seulement le texte mais encore le domaine spécialisé traité, avec ses modes de pensées spécifiques (Lundquist 2008).

C'est dans cette optique textuelle, véritablement dynamique et procédurale, que nous introduisons la catégorisation ci-dessous en discours, genre et type de texte dont la connaissance, et la reconnaissance, chez le lecteur (apprenant ou non) fonctionnera d'en haut, sous la forme d'hypothèses de lecture lui permettant de se frayer un chemin à travers le texte. Le lecteur le fera en segmentant, d'en bas, les éléments du texte arrivant, en unités linguistiques, des unités de lecture, dont la délimitation dépend entièrement de ses compétences de lecture, fondées sur ses connaissances du monde, des textes et de la langue en général ; et sur les domaines spécifiques traités par les textes sur objet spécifique en particulier. Tout en tirant sur des catégories textuelles comme déclencheurs d'attentes descendants et sur des unités linguistiques ascendantes pour identifier et vérifier, ou falsifier, ces attentes, la perspective procédurale fait que ces catégories soient essentiellement flottantes et les unités de nature mobile et flexible, ce qui ne les empêche pas de fonctionner parfaitement au niveau pratique tout comme au niveau théorique de la lecture.

\section{Catégories textuelles}

Nous partons du principe heuristique de «la détermination du global sur le local » (Rastier ce numéro), et plus spécifiquement, de modèles cognitifs et

2 Comme initié par Dressler (1972) et Coseriu (1981).

3 Chez par exemple Maingueneau (1976).

4 Halliday et Hasan (1976) parlent de texte, et Grimes (1975) de discours.

5 Exemplifiés par les «textes académiques» dans Lundquist (2013). 
psycholinguistiques, français (Denhière et Baudet 1992) comme anglo-saxons (Kintsch 1998), selon lesquels la lecture, dont le but ultime est la construction incrémentale d'une représentation mentale du contenu du texte, doit être saisie et décrite dans son processus réel d'interprétation en ligne du matériel linguistique arrivant. Suivant la linguistique cognitive, l'interprétation d'un texte se fait comme un va et vient entre des connaissances agissant d'en haut, à savoir des connaissances du monde et de textes, et des connaissances d'en bas de la, ou des langues. Dans notre perspective didactique et contrastive de la lecture en FLE, l'assomption cruciale est que les lecteurs-apprenants lisant un texte en langue étrangère possèdent déjà des savoirs fondamentaux, sur le monde, sur les textes, et sur la langue en général, celui-ci de par leur « instinct de langue », instancié dans leur langue maternelle. Ces savoirs sont organisés et structurés de manière identique à travers les langues, à un niveau très abstrait, il est vrai, dans des schémas cognitifs, qui comprennent, pour les textes, des connaissances des trois catégories discours, genre et type de texte; et, pour la langue, des connaissances sur la linéarisation des éléments linguistiques dans la phrase (i.e., l'ordre des mots canonique), et la valence verbale.

Nous posons donc qu'il y a des aspects partagés, communs à toute lecture de textes sur objets spécifiques, à travers les langues. Ainsi, il y a tout lieu de croire que tous lecteurs possèdent des schémas cognitifs, qui - à un certain niveau d'abstraction - sont identiquement structurés à travers des cultures, parce que fondés sur des expériences humaines communément partagées; par exemple de type aristotélicien, comme les relations événementielles de avant/après, causales de cause/conséquence, et de relations hypero-/hyponymiques, etc.

En ce qui concerne le niveau du texte, tout lecteur s'initiant à des textes académiques, s'est déjà familiarisé avec un éventail considérable de discours, de genres et de types de texte divers ; bien que les textes puissent revêtir des formes divergentes dans des langues et des cultures différentes, on peut supposer qu'il y a des structures de bases textuelles communes à toutes les cultures contemporaines. Mentionnons le discours familial et scolaire; les genres de fiction comme le roman et la poésie, et de non-fiction comme l'article de journal et le manuel scolaire, tout comme de toute probabilité, il existe des types de texte universels comme les types narratif, descriptif, expressif, etc.

Notre catégorisation de textes en discours (Lundquist 2013 : 20-22), se fonde sur la conception que propose Michel Foucault du discours comme «système anonyme de fonctionnement », tels le discours scientifique qui serait, selon lui, " composé de la définition d'un domaine d'objets, d'un ensemble de méthodes, d'un corpus de propositions considérées comme vraies, d'un jeu de règles et de définitions, de techniques et d'instruments » (Foucault 1971 : 23) ; ou le discours 
didactique " engendré par le système social de l'enseignement, et dont l'optique est de créer des compétences et aptitudes parmi les citoyens » (Lundquist 2013 : 20, inspiré par Luhman 1995). La catégorisation des textes en genres, tels par exemple le livre d'enseignement, l'article scientifique, le rapport et autre documentation technique et administrative, se fait selon la constellation de traits récurrents, respectant un ensemble de conventions socio-culturelles de production et de réception, ce qui fait surgir des attentes génériques. Et, finalement, notre classification de textes en types de texte suit de plus près l'agencement linguistique dominant le texte, aboutissant à huit types de base ou prototypiques : informatif, explicatif, d'exposition, argumentatif, expressif, narratif, descriptif et directif. ${ }^{6}$

Si les catégorisations ci-dessus saisissent des traits communs à travers les langues et les cultures, ce qui diverge entre des textes en L1 et L2, c'est évidemment le lexique, la syntaxe et la textualisation particuliers à une langue, c'est-à-dire la manière dont le lexique encode du sens, dont la syntaxe articule le contenu et présente de l'information, et dont les normes de textualisation propres à une langue - et à une culture - enchaînent et agencent l'information dans le déroulement d'un texte.

Pour les textes qui nous intéressent ici, les textes sur objet spécifique en français, les choses se compliquent du fait qu'il ne s'agit plus exclusivement de connaissances générales, et génériques, mais de savoirs spécialisés : un savoir (semi)-expert sur une partie du monde, tel un sujet particulier en philosophie, en histoire, en économie, etc. ; un savoir sur des discours spécialisés, tel le discours scientifique, un savoir sur des genres académiques comme l'article de recherche, et sur la langue «spécialisée », avec sa terminologie et ses formules particulières. Il n'empêche, comme le prédit la théorie cognitive de la lecture, que les connaissances déjà existantes soient primordiales pour acquérir des connaissances nouvelles, qu'il s'agisse de connaissances du monde ou d'une langue nouvelle. Il faut, pour ainsi dire, exploiter le savoir déjà existant du lecteur comme une passerelle pour accéder au savoir nouveau, et le savoir général pour accéder au savoir spécifique.

\section{Unités de lecture}

Si, pour les connaissances du monde et des textes, il y a ainsi déjà du savoir préacquis, c'est évidemment en ce qui concerne les unités linguistiques qu'il y a le

6 Pour une description détaillée de la classification de textes en discours, genre et type de texte, voir Lundquist (2008, 2013). 
plus d'obstacles à surmonter quand il s'agit de lire en langue étrangère. Or, nonobstant les différences qu'il puisse y avoir entre une L1 et une L2 au niveau du lexique et de la syntaxe, il existe chez tout lecteur un savoir linguistique général, "une grammaire innée ", bien que très souvent de manière inconsciente (Lundquist 2013 : 47). En toute langue, il faut mettre les mots en ligne dans un certain ordre, et en toute langue il y a des expressions qui renvoient à des personnes, choses et objets, et d'autres qui relatent des actions, événements et états ; et il y a des expressions qui mettent en place les acteurs sur la scène que constitue la phrase, et d'autres spécifiant les circonstances de lieu, de temps, de manière, etc.

Ces principes fondamentaux sont à exploiter dans le processus de la lecture en L2, selon la méthode didactique dite inter-compréhensive (Meissner et al. 2004 ; Lundquist 2013 : 48). Il faudra transmettre ces principes de L1 en L2 afin de pouvoir traiter les éléments linguistiques arrivant en ligne dans les phrases successives d'un texte en L2 selon ses règles grammaticales pour effectuer le "parsing » de la phrase, c'est-à-dire le groupement des éléments en constituants (GN, GV, etc.) et déterminer leur fonction syntaxique (sujet, verbe, objet, compléments) ainsi que leur rôle sémantique, AGENT, PATIENT, etc., mis en scène par le verbe principal de la phrase.

Considérant ces principes linguistiques généraux dans une perspective contrastive, le cas échéant comparant le danois comme L1 au français comme L2, il s'avère qu'il y a des différences fondamentales et systématiques entre ces deux langues (Baron et Herslund 2005), l'une appartenant au branche scandinave des langues germaniques, et l'autre aux langues romanes, différences qui s'accentuent dans les textes spécialisés en français, et qui risquent ainsi de poser problème au lecteur-apprenant danois pour son parsing en ligne des éléments entrants. Il s'agit tout d'abord du groupe nominal expansé en français, difficile à délimiter à droite pour un danophone qui s'attend à voir les adjectifs antéposés ; et d'autre part, il peut y avoir des problèmes à traiter les propositions "réduites », condensées à l'aide par exemple de formes verbales non-finies, qui sont d'une utilisation beaucoup moins fréquente en danois. Dans ce dernier cas, il s’agira de "désemballer», de "dépaqueter » le contenu. L'exemple ci-dessous illustrera ces deux types d'obstacles :

(1) L’incapacité des ministres des finances européens, réunis samedi 17 septembre à Wroclaw (Pologne), à se mettre d'accord sur la mise en œuvre du plan de secours à la Grèce décidé en principe le 21 juillet risque d'enflammer de nouveau les marchés. ${ }^{7}$

7 Le Monde. 20 septembre 2012. Cf. Lundquist (2013) : 80. 
La lecture exige l'acquisition et la maîtrise des règles de la langue française, et tout d'abord de ses règles pour présenter les mots dans un certain ordre et pour lier les mots en constituants, telles les règles d'accord, les prépositions et le schéma valenciel des verbes. Ce n'est qu'en maîtrisant ces règles que le lecteur saura tenir ensemble des mots en constituants, retarder la satisfaction de sa recherche du verbe principal ici jusqu'à la 36eme position dans la phrase (risque), et arriver à déterminer les fonctions syntactiques : le GN antéposé au VP étant le sujet, et le GV-non-fini postposé au VP l'objet direct. Il s'agit en même temps de « dépaqueter » l'information, de la déployer, des propositions condensées et des nominalisations, dont on peut compter une dizaine.

Il existe tout autant au niveau de la textualisation des différences systématiques d'une langue à l'autre, comme l'ont montré des études en linguistique textuelle contrastive. Comparant le danois et le français, nous avons trouvé des divergences au niveau des mécanismes locaux de cohésion textuelle, divergences engendrées par des différences lexicales, morphologiques et syntaxiques de la langue danoise comparée à la langue française. On peut mentionner la réalisation des relations anaphoriques - qu'elles soient infidèles (Lundquist 2004 ; Lundquist 2005; Lundquist 2006), résomptives (Lundquist 2007a ; Lundquist 2009) ou évolutives (Lundquist 2007b) - où le danois préfère, en règle générale, dans le cas non marqué, l'anaphorisation pronominale et le français l'anaphorisation nominale. L'anaphorisation pronominale correspondrait à une tendance générale à une structure de phrase endocentrique en danois, c'est-à-dire à une concentration du poids sémantique dans le verbe principal; et l'anaphorisation nominale à une structure exocentrique de la phrase française avec le poids sémantique dans les noms et groupes nominaux (Baron et Herslund 2005). Les relations rhétoriques aussi sont réalisées de manière différente en français et en danois ; en français, elles sont souvent réalisées par des propositions réduites dans des structures hypotactiques exploitant la morphologie non finie des verbes français (voir (1)). En danois, les mêmes faits et relations s'exprimeraient, en règle générale, dans des constructions paratactiques.

\section{Linguistique textuelle contrastive}

La linguistique textuelle contrastive a aussi son mot à dire sur l'organisation des idées dans des textes français comparés aux textes danois. Dans Lundquist (2014), nous avons montré, à partir d'analyses de textes 
appartenant au même discours (le discours scientifique-didactique), au même genre (le manuel scolaire), et au même type de texte (le type d'exposition), traitant le même objet spécifique (la sociologie), comment les textes français suivent un schéma "cartésien» et les textes danois un schéma "pragmatique », avec les caractéristiques respectives de leurs figurations textuelles, montrées dans Tableau 1.

Tableau 1: Deux types de figuration textuelle.

\begin{tabular}{ll}
\hline Figuration « cartésienne » en français & Figuration «pragmatique » en danois \\
\hline $\begin{array}{l}\text { Déduction à partir de principes généraux } \\
\text { Structure phrastique hiérarchique, hypotactique } \\
\text { Figurations argumentatives }\end{array}$ & $\begin{array}{l}\text { Induction à partir de la real life experience } \\
\text { Structure phrastique plate, paratactique } \\
\text { Figurations d'exposition et d'expression } \\
\text { subjective }\end{array}$ \\
$\begin{array}{ll}\text { Hypostatisation d'actions en faits (par les } \\
\text { nominalisations) }\end{array}$ & Mise en scène d'actions et d'acteurs \\
\hline \begin{tabular}{l} 
Abstraction éliminant actions et acteurs \\
\hline
\end{tabular}
\end{tabular}

\section{Unités flottantes}

La catégorie 'unité de lecture' peut sembler une unité ad hoc, subjective et élastique. Et elle l'est en effet. Elle dépend des capacités de lecture du lecteur(-apprenant), dont le scope, la portée visuelle autant que cognitive, dépend entièrement de son expérience ou manque d'expérience en lecture. Nous proposons toutefois de définir l'unité de lecture comme une unité de sens, délimitée selon trois critères : fonction, forme et frontières. La fonction de l'unité de lecture est avant tout cognitive : elle permet d'ouvrir l'accès à un domaine de savoir, à former des attentes et à commencer la construction d'un modèle mental, tout en y ajoutant progressivement des éléments. $L a$ forme de l'unité de lecture, c'est-à-dire sa nature (GN, GV, Gadv, etc.), sa morphologie (comme l'accord), et son rôle syntaxique ( $\mathrm{S}, \mathrm{V}$, O, etc.) permet d'en préciser son apport sémantique et cognitif, à la phrase et au texte. Finalement, les frontières de l'unité de lecture, à gauche comme à droite, sont déterminées par des faits perceptuels, comme la ponctuation et la position.

Tout en admettant que ces critères de définition ou de délimitation soient loin d'être stables, ils entrent parfaitement dans une approche procédurale de la dynamique même de la lecture qui oblige, il nous semble, à penser en catégories flexibles et souples. 


\section{Lecture raisonnée : catégories textuelles et unités de lecture}

Si nos catégories sont élastiques, d'autant plus il importe de montrer leur bien-fondé dans la perspective pratique et didactique qui est la nôtre ici. Nous reproduisons ci-dessous une lecture en ligne (Lundquist 2013 : 85-87) avec des hypothèses de lecture, à confirmer ou à corriger progressivement, en poursuivant la lecture. Ayant formulé ses hypothèses de lecture d'en haut, en regardant le texte et ses premiers signes, linguistiques comme extra-linguistiques (s'agit-il d'un livre qu'on tient dans ses mains? d'un article de recherche avec indication du journal scientifique dans lequel il a paru? le titre, qu'annonce-t-il ? etc.), le lecteur aura à se poser des questions concernant l'identification de chaque unité de lecture suivante, et de se formuler de nouvelles attentes. Cette lecture raisonnée comprend donc les opérations cognitives suivantes pour chaque unité :

- Identification : Qu'est-ce que c'est comme unité ? Quels éléments de sens peut-elle ajouter à mon modèle mental ?

- Attente : Qu'est-ce que je peux rencontrer par la suite?

- Vérification: l'unité que je lis maintenant, correspond-t-elle à ma prédiction?

- Correction : sinon, comment reformuler une prédiction de lecture?

Voici un (fragment de) texte à lire en ligne avec prédiction-identificationvérification-(correction) des unités de lecture, et avec la construction simultanée d'une représentation mentale du texte, primordiale pour la formulation de (nouvelles) hypothèses de lecture agissant d'en haut.

Catastrophes de grande ampleur. Les leçons du passé.

Organisation de coopération et de développement économiques

Depuis l'accident nucléaire de Tchernobyl jusqu'aux attaques terroristes du 11 septembre, en passant par l'ouragan Andrew ou le tremblement de terre de Kobe, les années récentes ont vu se succéder des catastrophes de grande ampleur qui ont causé de lourdes pertes en termes de vies, d'actifs et de richesse. Ces événements ont mis à mal le fonctionnement de systèmes vitaux tels que les transports ou l'approvisionnement en énergie, et eu des répercussions dans les zones avoisinantes et parfois même lointaines. Ils ont pu créer un sentiment général d'anxiété, et parfois entraîner une méfiance durable des citoyens quant à la capacité des gouvernants à les protéger. (OCDE 2005)

Dans Tableau 2, nous avons segmenté le texte en unités de lecture (colonne à gauche), en soulignant à des fins didactiques du FLE, les mots-outils, ou « mots 
Tableau 2: Exemple d'une lecture raisonnée.

Unités de lecture
Catastrophes de grande ampleur.
Les leçons du passé.
Organisation de coopération et de
développement économiques
Depuis l'accident nucléaire de
Tchernobyl

jusqu'aux attaques terroristes $d u 11$ septembre,

en passant par l'ouragan Andrew

les années récentes

ont vu

se succéder des catastrophes

de grande ampleur

\section{Lecture raisonnée}

Mes connaissances du domaine des grandes catastrophes sont activées.

Le mot leçons me fait prévoir un texte de type didactique, c'est-à-dire informatif, mais aussi directif, dans le genre de « quelles leçons peut-on - et doit-on tirer du passé ».

l'émetteur du texte est un organisme politicoéconomique international (OCDE).

depuis introduit une énumération temporelle (qui me fait attendre jusqu'à). l'accident nucléaire de Tchernobyl introduit la première catastrophe dans mon modèle mental. La phrase commence par une préposition, donc un complément circonstanciel, et non pas le sujet, habituellement à la première position. DONC : il faut retarder l'attente du sujet.

BINGO ! jusqu'à introduit la deuxième catastrophe attendue par l'énumération, et dont l'existence (mondialement) connue est signalée par le déterminant les $(a u x=\grave{a}+l e s)$; virgule signale petite pause, peutêtre continuation de l'énumération?

BINGO ! en passant par introduit un élément intermédiaire dans l'énumération, ici l'ouragan Andrew, déterminé par le.

ou le tremblement de terre de Kobe, la conjonction de coordination ou continue l'énumération de catastrophes. La virgule signale peutêtre la fin de l'élément circonstanciel antéposé ; si oui, je m'attends à voir le sujet grammatical de la phrase. constituant GN lié intérieurement par l'accord (féminin, pluriel), et qui pourrait jouer le rôle de sujet grammatical ; si oui, je m'attends à voir un GV au pluriel.

BINGO ! GV au pluriel, DONC : GN précédent est le sujet; le verbe principal voir me fait attendre un OD. BINGO ! OD trouvé, sous forme d'un infinitif suivi de son sujet « logique».

de ajoute un complément au nom catastrophes. 
Tableau 2: (continued)

\begin{tabular}{ll}
\hline Unités de lecture & Lecture raisonnée \\
\hline qui & $\begin{array}{l}\text { le pronom qui introduit une proposition relative, dans } \\
\text { laquelle il occupe la fonction de sujet ; je m'attends à } \\
\text { voir un GV au pluriel, } 3^{\text {ème }} \text { personne, s'accordant à } \\
\text { l'antécédent catastrophes. } \\
\text { BINGO ! VG, au pluriel, 3ème personne, identifié. Le } \\
\text { verbe principal causer me fait prévoir un OD. } \\
\text { ont causé } \\
\text { BINGO ! le OD trouvé. } \\
\text { de lourdes pertes } \\
\text { en termes de vies, } \\
\text { complément du nom pertes, par une précision en } \\
\text { termes de. La virgule me fait prévoir une coordination } \\
\text { d'actifs }\end{array} \quad$ BINGO ! premier élément coordonné. \\
et de richesse. & la conjonction et introduit le dernier élément de la \\
& coordination. Point final signale la fin de la phrase. \\
\hline
\end{tabular}

de liaison ", (en italique) qui, avec l'accord (en gras), contribuent justement à lier les mots entre eux en constituants. A droite, nous décrivons les étapes cognitives y correspondant, avec la notation 'BINGO !' pour les attentes satisfaites, et 'DONC :' pour les attentes à réviser.

\section{Conclusion}

Bien que les catégories textuelles - discours, genre et type de texte - flottent dans la tête du lecteur jusqu'au moment d'être activées lors de la lecture, c'est-àdire à l'encontre avec un texte particulier et son matériau linguistique (et extralinguistique) ; et bien que les unités linguistiques de lecture ne se figent et ne se déterminent qu'au moment d'entrer dans l'optique du lecteur et à l'encontre des attentes engendrées d'en haut, nous espérons avoir montré par notre exemple d'une lecture raisonnée ci-dessus, que catégories et unités ont une réalité cognitive essentielle et réelle. Elles sont en fait à définir et à identifier en raison de leur fonctionnalité concrète dans le processus de lecture, et selon la contribution qu'elles font en cours de route à l'élaboration d'une représentation mentale cohérente du contenu du texte. ${ }^{8} \mathrm{Si}$ les catégories et les unités

8 «La réalité des genres n'est donc pas a-temporelle et universelle. Ils n'existent que dans les événements socio-communicatifs singuliers que sont les textes et dans la reconnaissance sociocognitive des sujets producteurs et interprètes» (Adam 2013: 58). 
proposées dans le présent article sont ainsi flottantes, elles ont du moins le mérite de flotter dans la tête du lecteur, sous une forme plus ou moins consciente, au lieu de flotter dans l'air, dans l'abstraction pure et totale.

\section{Références}

Adam, Jean-Michel. 2013. Le paradigme du texte : Regard retrospectic et perspectives pour les sciences des textes. In M. Monte \& G. Philippe (éds.), Genres et textes, 297-325. Lyon : PUL.

Baron, Irène \& Michael Herslund. 2005. Langues endocentriques et langues exocentriques. Approche typologique du danois, du français et de l'anglais. Langue Française 145. 35-53.

Coseriu, Eugenio. 1981. Textlinguistik. Tübingen : Francke.

Denhière, Guy \& Serge Baudet. 1992. Lecture, compréhension de texte et science cognitive. Paris : PUF.

Dressler, Wolfgang. 1972. Einführung in die Textlinguistik. Tübingen : Francke.

Foucault, Michel. 1971. L'ordre du discours. Paris : Gallimard.

Grimes, Joseph E. 1975. The thread of discourse. Hague: Mouton.

Halliday, Michael A. K. \& Ruquaya Hasan. 1976. Cohesion in English. London: Longman.

Kintsch, Walter. 1998. Comprehension: A paradigm for cognition. New York: Cambridge University Press.

Luhmann, Niklas. 1995. Social systems. Stanford: Stanford University Press.

Lundquist, Lita. 2004. L'anaphore infidèle, regard(s) unificateur(s) sur le texte ?. In Dominique Klinger \& Sylvie Porheil (éds.), Regards croisés sur l'unité texte (actes du colloque de Chypre), 202-214. Pleyben : Association Perspective.

Lundquist, Lita. 2005. Noms, verbes et anaphores (in)fidèles: Pourquoi les Danois sont plus fidèles que les Français. Langue Française 145. 73-91.

Lundquist, Lita. 2006. Lexical Anaphors, information packing, and grammaticalisation of textual relations. In Henning Nølke, Irène Baron, Hanne Korzen, Iorn Korzen \& Henrik H Müller (éds.), Grammatica, 311-323. New York : Peter Lang.

Lundquist, Lita. 2007a. Academic discourse as social control and system(s), seen through the use of demonstrative noun phrases in French scientific texts. In Kjersti Fløttum (éd.), Language and discipline perspectives on academic discourse, 219-242. Newcastle : Cambridge Scholars Press.

Lundquist, Lita. 2007b. Comparing evolving anaphors in Danish and French. In Iørn Korzen \& Lita Lundquist (éds.), Comparing anaphors : Between sentences, texts, and languages, 111-125. Copenhagen : Copenhagen Business School.

Lundquist, Lita. 2008. Navigating in Foreign Language Texts. Copenhagen : Samfundslitteratur. Lundquist, Lita. 2009. L'anaphore résomptive : Navigation textuelle et comparaison discursive. In Federica Venier (éd.), Tra pragmatica e linguistica testuale ricordando Maria-Elisabeth Conte, 379-399. Alessandria : Edizioni dell'Orso.

Lundquist, Lita. 2013. Lire un texte académique en français. Paris : Ophrys.

Lundquist, Lita. 2014. «Cartesian French » and «Pragmatic Danes » : Language, text, cognition and culture. In Iørn Korzen, Angela Ferrari \& Anna-Maria De Cesare (éds.), Tra romanistica 
e germanistica : Lingua, testo, cognizione e cultura/Between Romance and Germanic : Language, text, cognition and culture, 315-334. Berne : Peter Lang.

Maingueneau, Dominique. 1976. Initiation aux méthodes de l'analyse du discours. Paris : Hachette.

Meissner, Franz-Joseph, Claude Meissner, Horst G. Klein \& Tilbert D. Stegmann (éds.). 2004. EuroComRom - Les sept tamis : Lire les langues romanes dès le départ. Aachen : Shaker Verlag.

OCDE. 2005. Catastrophes de grande ampleur : Les leçons du passé. Paris : Éditions OCDE. 